\title{
Sobrepeso, obesidad y salud percibida en contextos de pobreza de Tucumán, Argentina
}

\author{
Overweight, obesity and perceived health in contexts of \\ poverty in Tucumán, Argentina
}

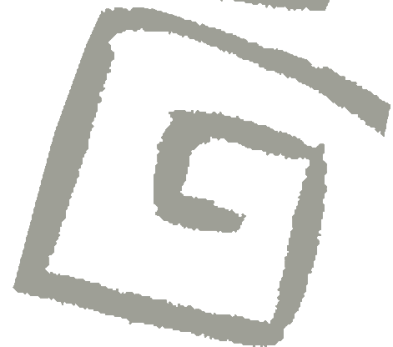

María Laura Cordero', María Florencia Cesani²

'Licenciada en Nutrición, Doctora en Ciencias Sociales. Becaria posdoctoral, Consejo Nacional de Investigaciones Científicas y Técnicas, con sede en Instituto Superior de Estudios Sociales. Tucumán, Argentina. $\square$ iD

2Licenciada en Antropología. Doctora en Ciencias Naturales. Investigadora independiente, Consejo Nacional de Investigaciones Científicas y Técnicas, con sede en Instituto de Genética Veterinaria, Facultad de Ciencias Veterinarias,

Universidad Nacional de La Plata. La Plata, Buenos Aires, Argentina. $\square$ (iD)
RESUMEN En contextos en los que la pobreza compromete la salud y la calidad de vida infantil, el estudio de la salud percibida permite identificar aspectos de la calidad de vida afectados por el sobrepeso y la obesidad. Desde esta perspectiva, los objetivos de este trabajo fueron: 1) determinar la prevalencia de sobrepeso y obesidad en escolares con edades comprendidas entre 8 y 12 años residentes en contextos de pobreza, 2) describir y comparar la percepción de la salud de niños y niñas según la presencia o ausencia de sobrepeso y obesidad, y 3) identificar eventuales factores asociados a la baja calidad de vida relacionada con la salud. Se realizó un estudio cuantitativo y transversal, en el que se aplicó el cuestionario KIDSCREEN-52 y se relevó el peso y la talla de 666 escolares residentes en el departamento de Simoca (provincia de Tucumán), el cual registra elevados porcentajes de pobreza estructural. Las altas prevalencias de sobrepeso $(23,0 \%)$ y obesidad $(27,2 \%)$ encontradas comprometen la percepción de la salud infantil, especialmente con relación a aspectos psicosociales, como el estado anímico, la autonomía, la aceptación social y los recursos económicos familiares.

PALABRAS CLAVES Sobrepeso; Obesidad; Salud Escolar; Calidad de Vida; Pobreza; Argentina.

\begin{abstract}
In contexts in which poverty compromises children's health and quality of life, the study of perceived health allows for the identification of aspects of quality of life affected by overweight and obesity. From this perspective, the objectives of this study were: 1 ) to determine the prevalence of overweight and obesity in schoolchildren aged 8-12 years living in contexts of poverty, 2) to describe and compare the perception of health of children according to the presence or absence of overweight and obesity, and 3) to identify possible factors associated with low quality of life related to health. A quantitative and cross-sectional study was conducted. The KIDSCREEN-52 questionnaire was applied and weight and height were assessed in 666 schoolchildren living in the department of Simoca (province of Tucumán), which has high percentages of structural poverty. The high prevalence of overweight (23.0\%) and obesity $(27.2 \%)$ affect the perception of health among children, especially in psychosocial dimensions such as moods and emotions, autonomy, social acceptance, and financial resources.
\end{abstract}

KEY WORDS Overweight; Obesity; School Health; Quality of Life; Poverty; Argentina. 


\section{INTRODUCCIÓN}

La condición de pobreza de una persona involucra un determinado grado de privación que impide el pleno desarrollo de sus capacidades $y$, en última instancia, de su libertad $^{(1,2)}$. Debido a que la pobreza es un constructo multidimensional, su definición y su delimitación resultan muy complejas ${ }^{(1,3)}$. Si bien existen diferentes enfoques y perspectivas para su análisis, en este trabajo nos referimos a la pobreza como la situación estructural en que se encuentra un conjunto de hogares, que le confiere el acceso limitado a determinados recursos, bienes y servicios materiales y que condiciona su inserción en la estructura socioproductiva ${ }^{(4)}$. Este concepto se encuentra atravesado por tres matices diferentes: la idea de carencia de recursos, bienes y servicios; de insatisfacción de aquellas necesidades que un grupo social satisface en su globalidad; y de marginalidad o exclusión de aquellos recursos, bienes y servicios que pueden compartirse en una sociedad ${ }^{(3)}$.

Se ha demostrado que la pobreza influye negativamente en el crecimiento, desarrollo y bienestar físico, cognitivo y socioemocional infantil ${ }^{(5,6)}$. Aspectos tales como los bajos ingresos familiares, la vivienda inconveniente y la inseguridad alimentaria repercuten en la salud ocasionando no solo la transmisión y perpetuación de enfermedades agudas, sino también crónicas ${ }^{(7)}$.

Entre las enfermedades crónicas de mayor prevalencia global se encuentra la obesidad. La Organización Mundial de la Salud ha informado recientemente que la ocurrencia de exceso de peso (sobrepeso + obesidad) entre los lactantes, niños y adolescentes va en aumento en todo el mundo, siendo los países de ingresos bajos y medios los que se ven más afectados ${ }^{(8)}$. Con relación a la asociación entre exceso de peso y pobreza se ha señalado que es variable y compleja ${ }^{(9,10)}$. Especialmente, en el contexto latinoamericano, autores como Peña y Bacallao afirman que el sobrepeso y la obesidad resultan cada vez más frecuentes en contextos carencia${ } \mathrm{es}^{(9)}$. En Argentina, estudios poblacionales han informado elevadas prevalencias de exceso de peso infantil para la macrorregión denominada Norte Grande Argentino ${ }^{(11,12)}$ -conformada por las provincias de Jujuy, Salta, Catamarca, Tucumán, Santiago del Estero, Chaco, Formosa, Corrientes y Misiones- señalada como la más postergada del país en términos sociales y económicos ${ }^{(13)}$. En dichos estudios, la prevalencia media reportada de obesidad infantil $(4,5 \%)$ COrrespondió en un $42 \%$ de los casos a niños pertenecientes a hogares con indicadores de indigencia y sin cobertura de las necesidades básicas. Datos más recientes, aportados por el Ministerio de Salud de la Nación ${ }^{(14)}$ evidenciaron que el 5,5\% de niños obesos menores de 5 años de la región del Noroeste Argentino pertenecían a familias con necesidades básicas insatisfechas. Asimismo, en el mismo informe se advierte que las prevalencias de exceso ponderal correspondientes a la provincia de Tucumán fueron del orden del $9,9 \%$ en varones y $8,1 \%$ en mujeres ${ }^{(14)}$.

Es conocido que, en ambientes empobrecidos, los niños están expuestos a peores condiciones de vida, menores recursos humanos de cuidado y dietas menos saludables ${ }^{(15)}$. En estos contextos, la estrategia familiar de consumo es invertir en volumen y no en calidad nutricional, predominando la ingesta de harinas, azúcares y grasas, que resultan más económicos y producen mayor saciedad ${ }^{(16)}$.

El sobrepeso y, especialmente, la obesidad infantil se asocian a una amplia gama de complicaciones de salud y a un creciente riesgo de contraer enfermedades prematuramente, entre ellas, diabetes y cardiopatías ${ }^{(8)}$. Además, dado que muchos problemas de salud en la vida adulta son consecuencia de alteraciones en el crecimiento y la malnutrición durante la infancia, el monitoreo de las primeras etapas de la trayectoria vital resulta de gran importancia ${ }^{(17)}$. En este sentido, tanto en el marco internacional como nacional se ha incrementado la preocupación por disponer de información acerca de las prevalencias de sobrepeso y obesidad en población infantil, especialmente, en grupos vulnerables $^{(14,18)}$. Asimismo, en estudios epidemiológicos se vienen empleando mediciones de 
salud percibida para identificar riesgos sanitarios y las múltiples dimensiones que pueden afectar la calidad de vida de diferentes grupos poblacionales, como consecuencia de una enfermedad o discapacidad. Desde la perspectiva de las políticas públicas, este tipo de estudios contribuye a determinar las necesidades en salud de cada población y permite proyectar el uso de los servicios sanitarios ${ }^{(19)}$.

La salud percibida o calidad de vida relacionada con la salud es un concepto subjetivo y multidimensional que describe de qué manera el estado de salud actual interviene en las capacidades individuales y permite alcanzar y mantener un nivel global de funcionamiento para la realización de actividades que resultan importantes y que repercuten en el bienestar ${ }^{(20)}$. Remite a dominios tanto propios (físicos y psicológicos) como externos al individuo (sociales), que interactúan con él y pueden modificar su estado de salud ${ }^{(21)}$.

En Argentina, investigaciones realizadas en poblaciones infantiles han informado que la salud percibida es sensible a aspectos individuales tales como el sexo, la edad y la presencia de enfermedad o discapacidad, así como también sociales ${ }^{(22,23,24)}$. Resulta de especial interés un estudio realizado en escolares de la ciudad de Bahía Blanca, en el que se observó que los niños y las niñas con diagnóstico de obesidad percibían peor su salud respecto de aquellos que no presentaban exceso de peso ${ }^{(24)}$. Por otra parte, en el mismo estudio la autora plantea que la presencia de sobrepeso y obesidad afectó la calidad de vida relacionada con la salud de manera desigual según el nivel económico en los distintos grupos de edad y sexo. Sin embargo, no se cuenta con estudios nacionales que profundicen el estudio de la salud percibida en contextos de pobreza estructural.

Considerando que el ámbito de residencia condiciona la salud y la calidad de vida infanti( ${ }^{(6)}$ para este trabajo se ha seleccionado como área de estudio un departamento de la provincia de Tucumán que ha sido históricamente reseñado por la persistencia de condiciones de vida desfavorables y que registra los mayores porcentajes provinciales de pobreza ${ }^{(25,26,27)}$. Por otra parte, se trata de un territorio que no dispone de información acerca de la situación nutricional de la población infantil. Es por ello que este trabajo tiene por objetivos: 1) determinar la prevalencia de sobrepeso y obesidad en escolares con edades comprendidas entre 8 y 12 años, residentes en contextos de pobreza; 2) describir y comparar la percepción de la salud de niños y niñas según la presencia o ausencia de sobrepeso y obesidad; y 3) identificar eventuales factores asociados a la baja calidad de vida relacionada con la salud en esta población.

Este trabajo se enmarca en el proyecto de tesis doctoral titulado "Calidad de vida y estado nutricional: sus manifestaciones en escolares de ámbitos urbanos y rurales de Tucumán", Facultad de Filosofía y Letras, Universidad Nacional de Tucumán (Resolución 702-144-2015).

\section{MATERIALES Y MÉTODOS}

\section{El ámbito de la investigación: Simoca como contexto de pobreza estructural}

El departamento de Simoca, ubicado en el sur de la provincia de Tucumán y a $50 \mathrm{~km}$ de la capital $\left(27^{\circ} 15^{\prime} 47^{\prime \prime} \mathrm{S} 65^{\circ} 21^{\prime} 28^{\prime \prime} \mathrm{O}\right)$, presenta una extensión de $1.261 \mathrm{~km}^{2}(5,6 \%$ de la superficie provincial) y alberga al $2,13 \%$ (30.876) de sus habitantes que, en su mayoría, residen en áreas rurales $(76,23 \%)^{(28)}$ (Figura 1).

En cuanto a sus características físicas, se encuentra en una amplia planicie aluvial atravesada por una densa red hidrográfica de ríos y arroyos ${ }^{(29)}$. Gran parte de su extensión presenta suelos salitrosos con terrenos bajos, poca permeabilidad y áreas propensas al anegamiento ${ }^{(30)}$, situación que agudiza la vulnerabilidad de sus residentes que anualmente sufren inundaciones ${ }^{(31,32,33)}$. Además, las tierras salinas dificultan la producción agrícola y ganadera, razón por la cual solo el área oeste del departamento se integra a las áreas de producción primaria ${ }^{(34)}$.

Es considerada una "sociedad rural tradicional", con una fuerte raigambre cultural y costumbres que se remontan al siglo 


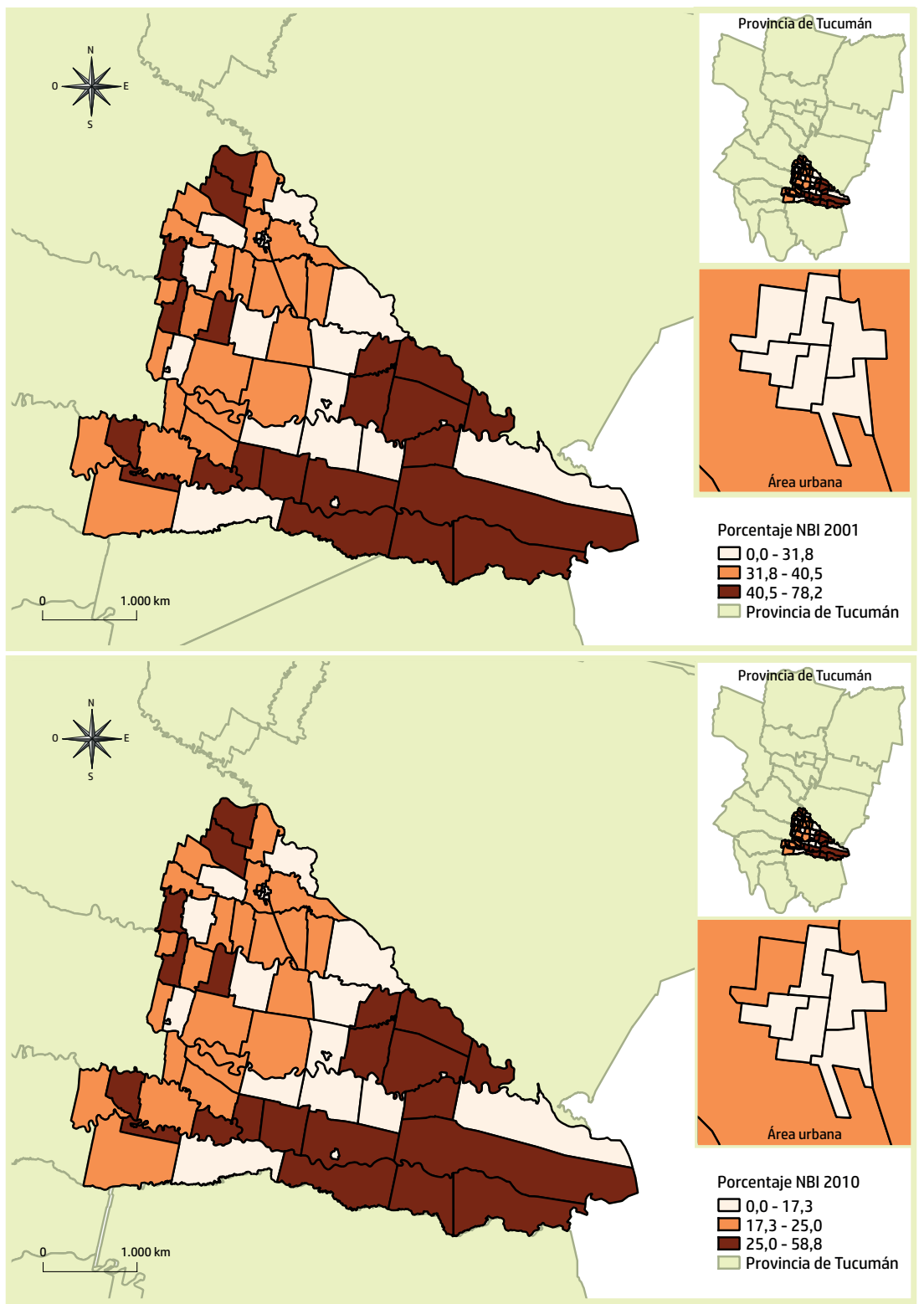

Figura 1. Distribución espacial de las necesidades básicas insatisfechas, por radio censal. Departamento de Simoca, provincia de Tucumán, Argentina. Periodo 2001-2010.

Fuente: Elaboración propia con base en datos del Censo Nacional de Población, Hogares y Viviendas, 2001-2010.

XVIII, cuando se funda oficialmente como comuna rural $^{(35)}$. Su población se distribuye en poblados aislados con baja densidad y características ambientales y socioculturales compartidas (ausencia de servicios e infraestructura básica, bajo nivel educativo, escaso desarrollo ocupacional y económico, etc.). La mitad de sus habitantes depende del empleo público, en tanto que el sector privado tiene escaso desarrollo ${ }^{(36)}$. Es quizás vinculado a ello que, como resultado de la migración de los grupos poblacionales económicamente activos a núcleos urbanos con mayor oportunidad de educación y empleo, desde 1980 su crecimiento poblacional ha sido predominantemente negativo ${ }^{(37)}$. 
Históricamente, Simoca registra porcentajes elevados de pobreza evaluados a partir del índice de necesidades básicas insatisfechas $(\mathrm{NBI})^{(26,27)}$. El estudio y seguimiento de este índice presenta como ventaja su carácter multidimensional, a la vez que permite la identificación de situaciones estructurales, ya que toma en cuenta indicadores que varían a largo plazo e implican al hacinamiento, la vivienda inconveniente, las condiciones sanitarias, la asistencia escolar y la capacidad de subsistencia. Desde esta perspectiva de análisis, se considera pobre al hogar que presenta al menos una de las carencias mencionadas. Por otra parte, la información sobre NBI se encuentra disponible con continuidad en las diferentes series censales nacionales y está desagregada al mínimo nivel administrativo, el radio censal (que contiene 300 viviendas) posibilitando una mayor precisión sobre su distribución espacial.

La evolución de las NBI en Tucumán evidencia que Simoca se ha ubicado por encima del percentil 75 durante los periodos censales 2001 y 2010, en el tercer y quinto puesto de mayor pobreza, respectivamente. Al indagar el comportamiento de este índice en el interior del departamento, en el año 2001, en la mitad de los radios censales, más del 37,4\% de los hogares se encontraba en situación de pobreza, siendo este porcentaje un $150 \%$ superior al promedio provincial. En aquel entonces, se detectaban unidades censales con incluso un $78,2 \%$ de hogares pobres, lo que representa una brecha de 70 puntos respecto de las áreas con menor carencia ${ }^{(27)}$. Aunque es de destacar que el último censo nacional del año 2010 evidenció un descenso en la media departamental de las NBI, puesto que la mitad de las unidades censales presentó alrededor del $21,8 \%$ de hogares pobres, la brecha sigue siendo amplia y su posición relativa en el contexto provincial permanece desfavorable. La persistencia de estas condiciones puede advertirse en la Figura 1, que muestra la espacialización de las NBI en los radios censales del departamento de Simoca para años 2001 y 2010.

\section{Diseño y población}

Se realizó un estudio cuantitativo y transversal en establecimientos educativos primarios públicos.

El relevamiento se realizó en las tres instituciones existentes en la zona urbana del partido y en cinco del sector rural, seleccionadas por conveniencia. La elección de estas últimas se basó en la matrícula escolar y la accesibilidad geográfica. Con relación a la matrícula escolar, cabe mencionar que Simoca reúne un total de 48 establecimientos educativos rurales. Según datos aportados por el Ministerio de Educación ${ }^{(38)}$, el $79 \%$ (38 escuelas) registraban -al momento del relevamiento- una matrícula baja (menor de 100 niños, de las cuales 20 escuelas tenían incluso menos de 50 niños, con un promedio de 38 niños correspondientes al rango etario considerado en este trabajo). El $21 \%$ de los establecimientos restantes (10 escuelas) presentaban una matrícula mayor de 100, con un promedio de 92 niños susceptibles de formar parte del estudio ${ }^{(38)}$. Sobre este último grupo de escuelas, se seleccionaron cinco, priorizando la accesibilidad. En este sentido, resulta pertinente mencionar que la mayoría de las escuelas rurales cuentan con un acceso sumamente precario, a través de caminos de tierra sin señalización y con servicio de transporte público irregular, de horarios restringidos y sujetos a condiciones climáticas. La presencia de precipitaciones persistentes en la región inhabilita dichos caminos por tiempo indeterminado, aislando a los poblados, por lo cual, el ausentismo es muy alto.

El ingreso a las escuelas fue gestionado ante las autoridades educativas competentes. La población elegible estuvo constituida por todos los niños de 8 a 12 años de edad escolarizados durante el ciclo lectivo 2015 (3.302 escolares $)^{(38)}$. Se excluyeron aquellos escolares que presentaron enfermedad manifiesta al momento del estudio, los que no contaron con el consentimiento informado de los padres o tutores y los que, aun teniéndolo, se rehusaron a participar. 
El estudio incluyó dos ejes de análisis: a) estudio antropométrico y b) estudio de la calidad de vida relacionada con la salud.

\section{Estudio antropométrico}

Fue realizado por una única antropometrista (María Laura Cordero), siguiendo las normas sugeridas por la Sociedad Argentina de Pediatría ${ }^{(39)}$. Se relevaron las siguientes variables: a) peso corporal $(\mathrm{P})(\mathrm{kg})$ : con el niño descalzo y vestido con ropa ligera, cuyo peso fue estimado y descontado del valor total registrado. Se empleó una balanza digital portátil Tanita UM-061 (precisión de $100 \mathrm{~g}$ ) y b) estatura total o talla (T) $(\mathrm{cm})$ : con el niño de pie, erguido y descalzo. Se midió sobre el plano sagital la distancia existente entre el vértex y el plano de apoyo. Se trabajó con un antropómetro vertical SECA (precisión de $1 \mathrm{~mm}$ ). En función de la fecha de nacimiento -obtenida del documento nacional de identidad de cada niño- se determinó la edad exacta (decimal) y a partir de los valores de $\mathrm{P}$ y $\mathrm{T}$ se calculó el índice de masa corporal $\left(\mathrm{IMC}=\left(\mathrm{P} / \mathrm{T}^{2}\right)(\mathrm{kg} /\right.$ $\left.\mathrm{m}^{2}\right)$, que fue ponderado con relación al sexo y la edad. Los casos de sobrepeso, obesidad y bajo IMC se determinaron empleando el programa informático WHO Antroplus v. $1.0 .3^{(40)}$ y teniendo en cuenta los siguientes puntos de corte: sobrepeso: valores de puntaje zIMC/edad $>1$ y $\leq 2$; obesidad: valores de puntaje zIMC/edad $>2$; bajo IMC: valores de puntaje zIMC/edad $<-2)^{(17)}$. Solo se registraron dos casos de bajo IMC que fueron excluidos de cualquier tipo de análisis. Así, quedaron definidos tres grupos de acuerdo con el estado nutricional antropométrico: 1) niños con sobrepeso (S); 2) niños con obesidad $(\mathrm{O})$ y 3 ) niños sin exceso de peso: aquellos que presentaron valores de zIMC/edad $>-2$ y $<1$ ).

\section{Estudio de la calidad de vida relacionada con la salud}

Se aplicó la versión para niños del cuestionario internacional KIDSCREEN-52, adaptado y validado para la población argentina ${ }^{(23,41)}$.
Constituye el primer instrumento de calidad de vida relacionada con la salud desarrollado simultáneamente en varios países para uso en la población infantil y adolescente de 8 a 18 años, y elaborado a partir de los propios discursos sobre salud y bienestar, mediante grupos focales y técnicas psicométricas de selección de ítems ${ }^{(42)}$. El cuestionario informa sobre diez dimensiones de salud percibida, definidas por el grupo Kidscreen ${ }^{(42)}$ de la siguiente manera:

1. Bienestar físico: considera los niveles de actividad física, energía y condición física del niño.

2. Bienestar psicológico: implica las emociones positivas y la satisfacción con la vida.

3. Estado de ánimo y emociones: abarca las experiencias negativas, los estados de ánimo depresivos y sensaciones de estrés.

4. Autopercepción: la imagen corporal es explorada por las cuestiones relativas a la satisfacción con la apariencia, al igual que con la ropa y otros accesorios personales.

5. Autonomía: refiere a la libertad de elección, autosuficiencia e independencia del niño. Implica, por otra parte, si el niño considera que posee oportunidades para participar en actividades sociales, especialmente actividades de ocio y pasatiempos.

6. Relación con los padres y vida familiar: refiere a la calidad de la interacción y sentimientos entre el niño y sus padres o cuidadores.

7. Relación con los amigos y apoyo social: involucra la naturaleza de las relaciones con otros niños (amigos y compañeros), en cuanto a la calidad de la interacción y el apoyo percibido.

8. Entorno escolar: incluye la satisfacción con su capacidad y desempeño escolar.

9. Aceptación social: recoge los sentimientos de rechazo por parte de los compañeros de escuela, así como también la sensación de ansiedad hacia estos.

10. Recursos económicos: describe si el niño siente que tiene suficientes recursos que le permitan un estilo de vida semejante al de otros niños. 
Las respuestas a los 52 ítems que incluye la encuesta se categorizaron en una escala de tipo Likert de cinco opciones que evaluó la frecuencia o la intensidad del atributo con un período recordatorio de una semana (siempre, casi siempre, a veces, casi nunca y nunca o nada, un poco, moderadamente, mucho o muchísimo).

Para la administración del cuestionario, se acondicionó un salón en cada escuela, a los fines de minimizar las distracciones. La presentación tuvo una duración aproximada de cinco minutos, mientras que los niños y las niñas lo completaron en un rango de entre 20 y 60 minutos, requiriendo de mayor tiempo y asistencia los y las escolares de edades menores (8 y 9 años) o con dificultades de lectoescritura. En estos casos, una licenciada en psicología asistió a las niñas y los niños, resguardando la confidencialidad de las respuestas ofrecidas. En todos los casos se siguieron las recomendaciones de administración del cuestionario propuestas por el grupo Kidscreen ${ }^{(42)}$.

\section{Análisis estadístico}

Para el análisis estadístico se empleó el programa SPSS versión 19.0. A fin de detectar datos potencialmente incorrectos, se realizó el control de la calidad de la base de datos siguiendo el protocolo de revisión sugerido por la Organización Mundial de la Salud ${ }^{(40)}$. Por cuanto no se detectaron valores de puntaje z IMC/edad mayores a 5, no fue necesario excluir ningún caso.

Se calcularon prevalencias generales de sobrepeso y obesidad en ambos sexos. Para cada dimensión de la encuesta de calidad de vida relacionada con la salud se calcularon puntajes Rasch que posteriormente fueron estandarizados a una media (M) de 50 y a un desvío estándar (DE) de 10. Mayores puntajes indican una mejor calidad de vida relacionada con la salud $(M+1 D E)$, en tanto que puntajes menores a 40 (M-1DE) implican baja calidad de vida relacionada con la salud o su compromiso ${ }^{(42)}$.
Para comparar la percepción de la salud según la presencia o ausencia de sobrepeso y obesidad se efectuaron análisis de varianza (ANOVA) y pruebas post hoc de diferencia mínima significativa (DMS), considerando un nivel de significación de $p<0,05$.

Para analizar el efecto del sobrepeso y la obesidad sobre la calidad de vida relacionada con la salud se estimó, para cada dimensión, diferencias de medias estandarizadas y se compararon, mediante pruebas $\mathrm{t}$ de Student, los grupos con sobrepeso y obesidad respecto del grupo sin exceso de peso (significación $\mathrm{p}<0,05$ ).

Finalmente, para identificar la asociación entre los factores sobrepeso, obesidad y sexo con la baja calidad de vida relacionada con la salud (dimensiones con puntajes iguales o inferiores a 40) se estimaron odds ratios (OR $)$. Se evaluó la asociación con el test de Wald y en aquellas dimensiones en las que $p \leq 0,10$, se ajustó un modelo de regresión logística multivariada, estimándose odds ratios ajustadas $\left(\mathrm{OR}_{\mathrm{a}}\right)$ y sus intervalos de confianza con un nivel del 95\% (IC 95\%). Las OR constituyen una medida de efecto de uso en estudios transversales que permite aproximar la posibilidad de ocurrencia de un evento. Los límites del intervalo de confianza reflejan el tamaño del efecto mínimo y máximo asociado a una determinada exposición ${ }^{(43)}$.

\section{Consideraciones éticas}

Para este estudio se solicitó el consentimiento y la autorización escrita de padres o tutores de los niños y las niñas participantes. Además, al momento del relevamiento antropométrico y de la realización de la encuesta se solicitó el asentimiento de los y las escolares. La investigación se desarrolló conforme a las recomendaciones nacionales e internacionales sobre investigación humana. El protocolo de este estudio fue aprobado por el Comité de Ética e Investigación del Departamento de Epidemiología del Sistema Provincial de Salud de Tucumán. 


\section{RESULTADOS}

De la matrícula correspondiente a niños de 8 a 12 años (797), consignada en los registros de las escuelas incluidas en este estudio, 123 niños no fueron autorizados por sus padres o tutores, se rehusaron a participar o estuvieron ausentes el día del muestreo. Se incluyeron en el análisis los datos antropométricos y las encuestas de un total de 666 niños y niñas (Tabla 1), previa exclusión de cuatro protocolos con datos incompletos, correspondientes a dos niños con bajo IMC y dos con edades no contempladas en el estudio. Los casos considerados representaron el $20 \%$ de la matrícula escolar pública departamental ${ }^{(38)}$.

El análisis antropométrico indicó que el $50,2 \%$ de los escolares presentó exceso de peso: el $23,0 \%$ sobrepeso y el $27,2 \%$ obesidad. Las prevalencias calculadas con relación al sexo fueron del $23,9 \%$ con sobrepeso y el $27,9 \%$ con obesidad en niños, y del $22,0 \%$ con sobrepeso y el $26,4 \%$ con obesidad en niñas (Tabla 2).

En la Figura 2 se advierte que, al comparar las dimensiones de la calidad de vida relacionada con la salud entre los tres grupos de estado nutricional, aquellos sin exceso de peso puntuaron más alto que los grupos con sobrepeso y obesidad en la mayoría de las dimensiones, excepto en relación con los padres y vida familiar, relación con los amigos y apoyo social y entorno escolar, en las que los escolares con sobrepeso puntuaron más alto. No obstante, los resultados del ANOVA indicaron diferencias significativas solo para las dimensiones autopercepción $(\mathrm{F}=4,186 ; p=0,016)$, autonomía $(\mathrm{F}=4,068$; $p=0,018)$ y recursos económicos $(\mathrm{F}=6,523$; $p=0,002)$. Los contrastes post hoc indicaron que dichas diferencias correspondieron a las siguientes comparaciones: autopercepción (sin exceso de peso-obesidad $p=0,008$ ); autonomía (sin exceso de peso-obesidad $p=0,005$ ) y recursos económicos (sin exceso de peso-sobrepeso $p=0,007$ y sin exceso de peso-obesidad $p=0,002$ ).

En la Figura 3 se presentan las puntuaciones medias e intervalos de confianza para las
Tabla 1. Composición de la muestra según edad y sexo de los escolares $(n=666)$. Departamento de Simoca, Tucumán, Argentina, 2015.

$\begin{array}{lccc}\text { Edad (años) } & \mathrm{N} & \text { Varones } & \text { Mujeres } \\ 8,0-8,99 & 176 & 91 & 85 \\ 9,0-9,99 & 137 & 67 & 70 \\ 10,0-10,99 & 188 & 95 & 93 \\ 11,0-11,99 & 165 & 95 & 70 \\ \text { Total } & 666 & 348 & 318 \\ \text { Fuente: Elaboración propia. } & & & \end{array}$

dimensiones de calidad de vida relacionada con la salud en varones y mujeres por separado, de acuerdo a su estado nutricional. En los varones, se observaron diferencias significativas entre los tres grupos para las dimensiones estado de ánimo y emociones $(\mathrm{F}=3,367 ; p=0,027)$, autopercepción $(\mathrm{F}=3,529 ; p=0,030)$ y recursos económicos $(\mathrm{F}=4,923 ; p=0,008)$. Los contrastes post hoc indicaron que dichas diferencias correspondieron a las siguientes comparaciones: estado de ánimo y emociones (sin exceso de peso-sobrepeso $p=0,021$ y sin exceso de peso-obesidad $p=0,037$ ), autopercepción (sin exceso de peso-obesidad $p=0,011$ y sobrepeso-obesidad $p=0,050$ ) y recursos económicos (sin exceso de peso-sobrepeso $p=0,018$ y $\sin$ exceso de peso-obesidad $p=0,006)$. Por su parte, en las mujeres, la única dimensión que evidenció diferencias significativas entre los grupos de estado nutricional fue autopercepción ( $\mathrm{F}=3,426 ; p=0,034)$, que correspondió, fundamentalmente, a diferencias entre los

Tabla 2. Prevalencias generales (\%), número de casos e intervalos de confianza del estado nutricional en niños y niñas. Departamento de Simoca, Tucumán, Argentina, 2015.

\begin{tabular}{|c|c|c|c|c|c|c|c|}
\hline \multirow[t]{2}{*}{ Estado nutricional } & \multicolumn{2}{|c|}{$\begin{array}{c}\text { Niños } \\
(n=348)\end{array}$} & \multicolumn{2}{|c|}{$\begin{array}{c}\text { Niñas } \\
(n=318)\end{array}$} & \multicolumn{2}{|c|}{$\begin{array}{c}\text { Total } \\
(n=666)\end{array}$} & \multirow[t]{2}{*}{ IC 95\% } \\
\hline & $n$ & $\%$ & $n$ & $\%$ & $n$ & $\%$ & \\
\hline Sin exceso de peso & 168 & 48,3 & 164 & 51,6 & 332 & 49,8 & $46,04-53,66$ \\
\hline Sobrepeso & 83 & 23,9 & 70 & 22,0 & 153 & 23,0 & $19,77-26,18$ \\
\hline Obesidad & 97 & 27,9 & 84 & 26,4 & 181 & 27,2 & $23,79-30,56$ \\
\hline
\end{tabular}




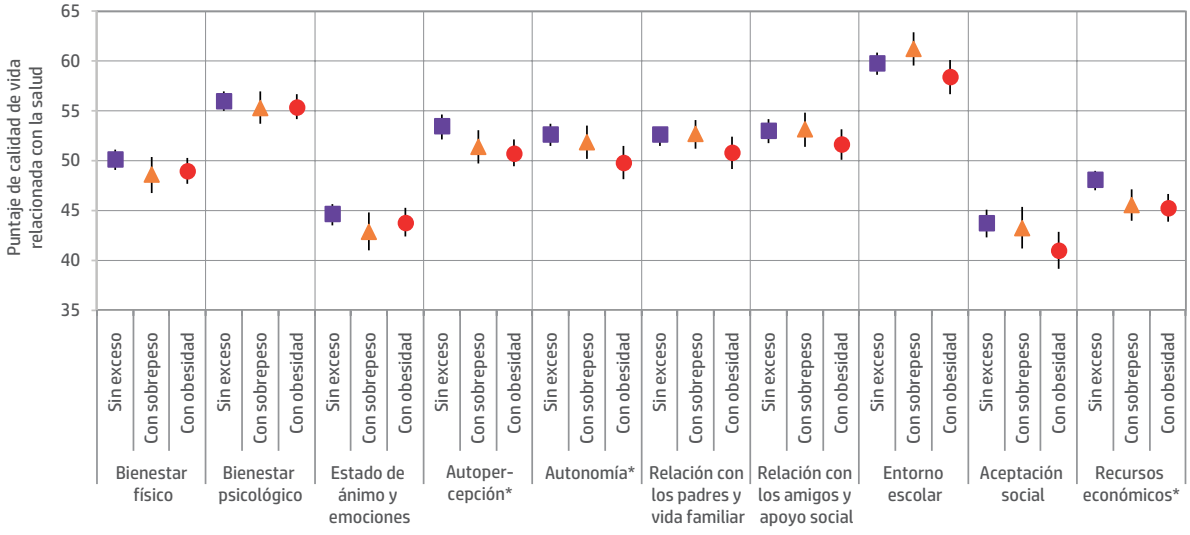

Figura 2. Puntuaciones medias e intervalos de confianza para las dimensiones de calidad de vida relacionada con la salud según categorías de estado nutricional (sin exceso de peso, con sobrepeso, con obesidad) en niños y niñas. Departamento de Simoca, Tucumán, Argentina, 2015.

Fuente: Elaboración propia

* Diferencias estadísticamente significativas $(p<0,05)$. Análisis de varianza (ANOVA).
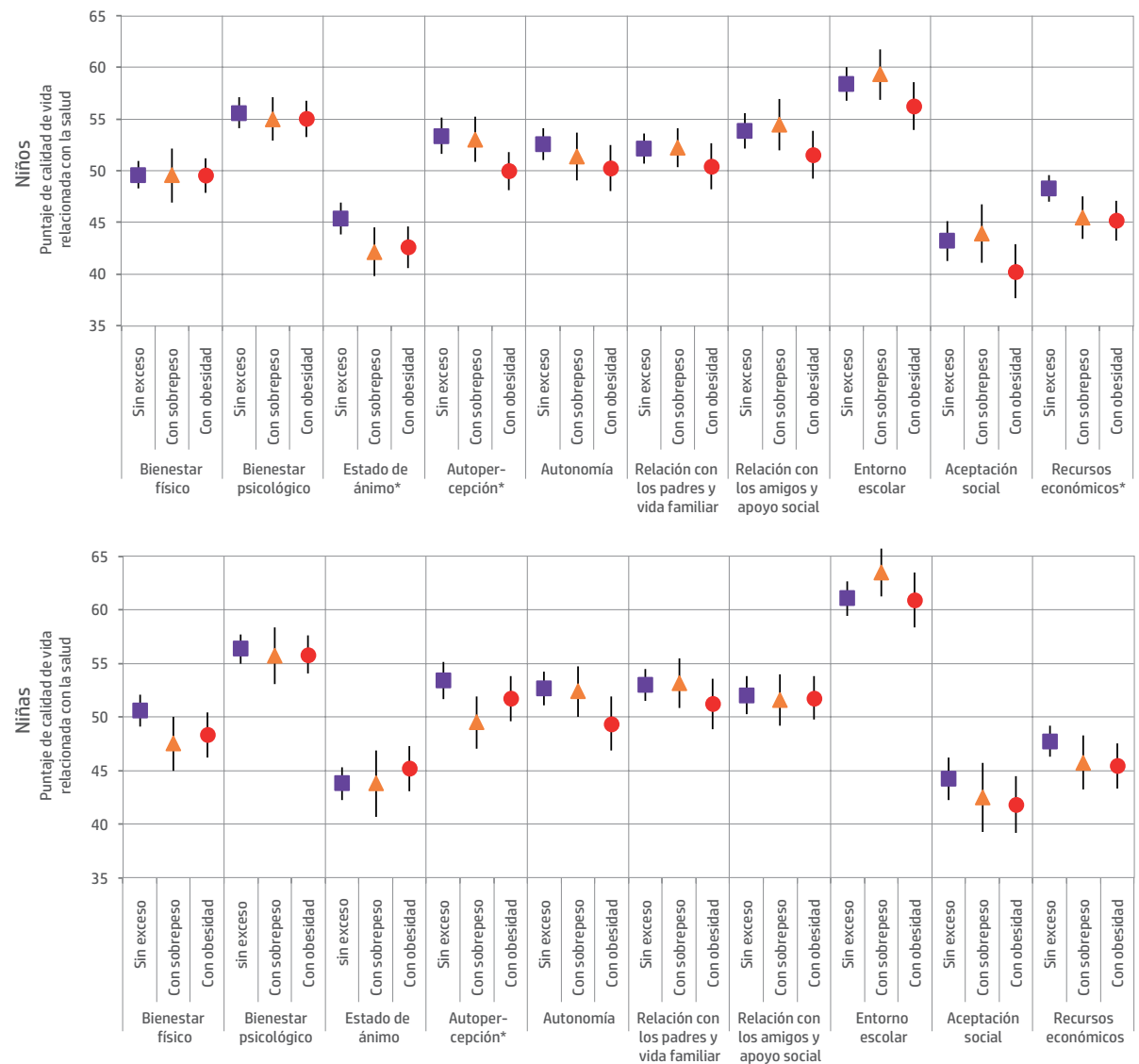

Figura 3. Puntuaciones medias e intervalos de confianza para las dimensiones de calidad de vida relacionada con la salud según categorías de estado nutricional (sin exceso de peso, con sobrepeso, con obesidad) en niños y niñas. Departamento de Simoca, Tucumán, Argentina, 2015. 
escolares sin exceso de peso y aquellos con sobrepeso $(p=0,010)$.

Por último, se verificó mediante análisis multivariado que, tanto el sobrepeso como la obesidad se asociaron a una percepción negativa de los recursos económicos, en tanto que el sobrepeso lo hizo con el bajo estado de ánimo y emociones y la obesidad con la baja autonomía y la baja aceptación social. Por el contrario, no se encontró asociación entre el sexo y las dimensiones incluidas en este análisis (Tabla 3).

Tabla 3. Odds ratios crudas y ajustadas para las dimensiones de calidad de vida relacionada con la salud según estado nutricional y sexo. Departamento de Simoca, Tucumán, Argentina, 2015.

\begin{tabular}{|c|c|c|c|c|c|c|c|c|}
\hline \multirow{3}{*}{ Dimensión } & \multicolumn{5}{|c|}{ Análisis bivariado } & \multicolumn{3}{|c|}{ Análisis multivariado } \\
\hline & \multicolumn{2}{|c|}{ Baja CVRS } & \multirow[t]{2}{*}{ ORc } & \multirow[t]{2}{*}{ IC $95 \%$} & \multirow[t]{2}{*}{ Valor de $p$} & \multirow[t]{2}{*}{ ORa } & \multirow[t]{2}{*}{ IC $95 \%$} & \multirow[t]{2}{*}{ Valor de $p$} \\
\hline & $\mathrm{n}$ & $\%$ & & & & & & \\
\hline \multicolumn{9}{|c|}{$\begin{array}{l}\text { Estado de ánimo y } \\
\text { emociones }\end{array}$} \\
\hline \multicolumn{9}{|c|}{ Estado Nutricional } \\
\hline Sin exceso ${ }^{1}$ & 100 & 30,2 & - & - & - & - & - & - \\
\hline Sobrepeso & 62 & 40,8 & 1,58 & $1,09-2,30$ & $0,02^{*}$ & 1,59 & $1,07-2,38$ & $0,02^{*}$ \\
\hline Obesidad & 55 & 30,7 & 0,88 & $0,61-1,27$ & 0,49 & 1,03 & $0,69-1,52$ & 0,89 \\
\hline \multicolumn{9}{|l|}{ Sexo } \\
\hline Varones $^{1}$ & 112 & 51,6 & - & - & - & - & - & - \\
\hline Mujeres & 105 & 48,4 & 1,04 & $1,09-2,30$ & 0,81 & 1,05 & $0,76-1,45$ & 0,77 \\
\hline \multicolumn{9}{|l|}{ Autonomía } \\
\hline \multicolumn{9}{|c|}{ Estado Nutricional } \\
\hline Sin exceso ${ }^{7}$ & 24 & 7,3 & - & - & - & - & - & - \\
\hline Sobrepeso & 11 & 7,2 & 0,72 & $0,36-1,42$ & 0,72 & 0,98 & $0,48-2,11$ & 0,98 \\
\hline Obesidad & 26 & 14,5 & 2,18 & $1,27-3,73$ & $0,01^{*}$ & 2,19 & $1,22-3,96$ & $0,01^{*}$ \\
\hline \multicolumn{9}{|l|}{ Sexo } \\
\hline Varones $^{1}$ & 28 & 8,1 & - & - & - & - & - & - \\
\hline Mujeres & 33 & 10,4 & 1,32 & $0,78-2,25$ & 0,30 & 1,35 & $0,79-2,29$ & 0,27 \\
\hline \multicolumn{9}{|c|}{ Aceptación social } \\
\hline \multicolumn{9}{|c|}{ Estado Nutricional } \\
\hline Sin exceso ${ }^{7}$ & 144 & 43,5 & - & - & - & - & - & - \\
\hline Sobrepeso & 66 & 43,4 & 0,88 & $0,61-1,26$ & 0,48 & 0,99 & $0,68-1,46$ & 0,98 \\
\hline Obesidad & 94 & 52,5 & 1,44 & $1,02-2,02$ & $0,04^{*}$ & 1,48 & $1,02-2,14$ & $0,04^{*}$ \\
\hline \multicolumn{9}{|l|}{ Sexo } \\
\hline Varones $^{1}$ & 161 & 46,5 & - & - & - & - & - & - \\
\hline Mujeres & 143 & 45,3 & 0,95 & $0,70-1,30$ & 0,74 & 0,96 & $0,70-1,30$ & 0,77 \\
\hline \multicolumn{9}{|c|}{ Recursos económicos } \\
\hline \multicolumn{9}{|c|}{ Estado Nutricional } \\
\hline Sin Exceso ${ }^{1}$ & 73 & 22,1 & - & - & - & - & - & - \\
\hline Sobrepeso & 51 & 33,6 & 1,49 & $1,01-2,21$ & $0,04^{*}$ & 1,79 & $1,17-2,75$ & $0,02^{*}$ \\
\hline Obesidad & 56 & 31,3 & 1,31 & $0,91-1,92$ & 0,15 & 1,62 & $1,07-2,43$ & $0,01^{*}$ \\
\hline \multicolumn{9}{|l|}{ Sexo } \\
\hline Varones $^{1}$ & 90 & 26,0 & - & - & - & - & - & \\
\hline Mujeres & 90 & 28,5 & 1,13 & $0,80-1,59$ & 0,47 & 1,15 & $0,82-1,63$ & 0,42 \\
\hline
\end{tabular}




\section{DISCUSIÓN}

Las prevalencias de sobrepeso y obesidad encontradas en el presente estudio, y que involucran a la mitad de la población infantil de Simoca, resultan preocupantemente elevadas. Incluso superan a las informadas por otros autores que han realizado estudios similares en el ámbito nacional. Por ejemplo, Polleti y Barrios ${ }^{(44)}$ registraron un $21,6 \%$ de exceso de peso en escolares correntinos, en tanto que el estudio multicéntrico desarroIlado por Oyhenart et al. ${ }^{(45)}$ dio cuenta que Chubut fue la provincia con los porcentajes más altos $(26,7 \%)$, en contraste con otras como Catamarca y Jujuy que registraron prevalencias del orden del 10,8\% y $12,8 \%$, respectivamente. Estudios más recientes, efectuados en poblaciones escolares de las provincias de Santa Cruz, Buenos Aires y Mendoza, coinciden en informar porcentajes inferiores a los aquí hallados $22,46,47,48,49)$. La disparidad encontrada entre nuestros resultados y los de los mencionados estudios podría deberse, en parte, al uso de criterios metodológicos diferentes. En tal sentido, se ha puesto de relieve que las prevalencias de la malnutrición por exceso pueden variar significativamente en función de la referencia o estándar elegido para su diagnóstico ${ }^{(50,51)}$.

El aumento global de sobrepeso y obesidad infantil se atribuye a una multiplicidad de factores. Entre ellos, la alimentación y los estilos de vida se reconocen como los más importantes ${ }^{(52)}$. Estos, a su vez, se ven condicionados por el entorno, físico, económico, político y sociocultural. De allí la importancia de analizar el problema no solo a través del estudio de sus causas inmediatas, sino también de los factores subyacentes que pueden incidir sobre las anteriores ${ }^{(53)}$. Así, para comprender estos hallazgos en el contexto de la pobreza estructural, es posible pensar en las dimensiones simbólicas y culturales que podrían estar operando en las prevalencias de sobrepeso y obesidad encontradas. Según Aguirre $^{(54)}$ las representaciones culturales son construcciones, producto y sostén de las divisiones de clase, que se apoyan en el gusto para construir y justificar, en la vida cotidiana, una elección sesgada de alimentos, preparaciones y platos, con maneras y actitudes en la mesa, adecuadas a dichos consumos. En este sentido, se argumenta que cada sector de ingresos piensa al cuerpo, los alimentos y la comensalidad en forma diferente. Para los más pobres el cuerpo debe ser fuerte y para alimentarlo se requiere que la comida cumpla con esa búsqueda de fortaleza: los alimentos son rendidores ${ }^{(16)}$. En sociedades en las que la pobreza es persistente, como es el caso de Simoca, se podría pensar que la distribución del exceso de peso respondería a concepciones vinculadas a la abundancia y la salud infantil; más aun si se considera la fuerte connotación que su contracara (la desnutrición) tiene en Tucumán, luego de la exposición mundial de muertes infantiles por desnutrición ocurrida en la provincia, como consecuencia de la crisis socioeconómica ocurrida en el año 2001 en nuestro país ${ }^{(55)}$.

Pero además, las familias de bajos recursos se enfrentan a la dificultad de acceder a una alimentación balanceada energéticamente, ya que habitualmente los alimentos que tienen a su alcance son artículos de menor costo, industrializados, de producción masiva e indiferenciada (pan, fideos, azúcar, papas, carnes grasas, etc.), situación que lleva a estos grupos de población a seguir un patrón alimentario inadecuado, monótono y desbalanceado ${ }^{(15)}$.

Con relación a la salud percibida, los niños y las niñas con sobrepeso u obesidad presentaron una calidad de vida relacionada con la salud más baja que aquellos sin exceso ponderal. Solo al describir las apreciaciones referidas al contexto escolar, los y las escolares con sobrepeso lo percibieron mejor que sus pares normonutridos. Estos resultados coinciden con los previamente informados por otros autores que han encontrado una asociación negativa entre el IMC y la calidad de vida relacionada con la salud en poblaciones infantiles, especialmente en aquellos dominios relacionadas con aspectos físicos, emocionales y sociales ${ }^{(56,57,58)}$. Por otra parte, aunque las diferencias encontradas en la percepción de la salud en varones y mujeres con sobrepeso y 
obesidad, respecto de sus pares sin exceso de peso no se mantuvieron al efectuarse el análisis multivariado, se destaca que fueron los varones obesos quienes manifestaron mayor afectación de su calidad de vida relacionada con la salud.

A partir del análisis multivariado fue posible verificar que el autoreporte de bajo estado de ánimo fue más frecuente en los escolares con sobrepeso. Si bien merece la pena señalar que el cuestionario aplicado en este estudio no constituye una técnica diagnóstica, no debe perderse de vista que los niños con síntomas emocionales tienen mayor probabilidad de desarrollarlos en la vida adulta ${ }^{(59)}$. Al respecto, trabajos realizados en Argentina reportan que los contextos de pobreza suelen generar síntomas psicológicos ${ }^{(60,61,62)}$. Sin embargo, este fenómeno no se ha estudiado en profundidad en poblaciones infantiles y los resultados encontrados ameritan el desarrollo de líneas de investigación nuevas sobre aspectos psicosociales de la malnutrición por exceso en contextos de pobreza.

La etapa escolar es importante en el proceso de socialización, dado que constituye el periodo durante el cual el niño incorpora nuevos vínculos externos al círculo familiar y amplía su mundo de relación. Disponer de momentos de ocio y recreación resulta importante dado que constituye el sustrato en el que se van a configurar las primeras relaciones durante la infancia y adolescencia, lo que posibilita interacciones satisfactorias con otras personas en las etapas posteriores de la vida $^{(63)}$. En este sentido, cabe destacar que los niños y niñas obesos de Simoca sintieron más a menudo tener pocas posibilidades de crear y disponer de su tiempo de ocio y social. Reportaron, además, menor autosuficiencia e independencia y se sintieron más rechazados por sus compañeros que aquellos que no presentaban exceso de peso. Con relación a ello, se ha informado que es habitual que los niños obesos se sientan inferiores, rechazados o maltratados por sus amigos y compañeros $^{(64,65)}$. Además de los efectos psicológicos asociados al rechazo entre pares, se ha reseñado que quienes atraviesan estas situaciones presentan mayor posibilidad de problemas de integración y desempeño escolar ${ }^{(66,67)}$. Estos resultados ponen en evidencia la necesidad de realizar intervenciones integrales, que indaguen, desde un enfoque no solo cuantitativo, sino también cualitativo, los significados y representaciones que los niños con exceso de peso tienen de su propia salud.

Por último, en comparación con sus pares sin exceso de peso, los escolares con sobrepeso u obesidad reportaron baja apreciación de los recursos económicos disponibles en sus hogares. Si bien existe suficiente evidencia sobre los efectos de la pobreza sobre la calidad de vida relacionada con la salud desde la mirada de los adultos, pocos estudios han explorado la comprensión que tienen los niños de sus propias experiencias ${ }^{(68)}$.

Los resultados obtenidos aportan información novedosa y factible de ser utilizada en la planificación de políticas públicas que fomenten hábitos de alimentación y actividad física saludables y que mejoren la calidad de vida general de los niños de Simoca. Asimismo, la transferencia de estos resultados a espacios comunitarios locales, como escuelas, centros de salud, clubes y comunas favorecerá el abordaje integral del problema con el consecuente empoderamiento de los actores involucrados.

\section{CONCLUSIONES}

En los niños y las niñas de Simoca convergen condicionantes físicos (exceso de peso) y ambientales (pobreza) que condicionan su bienestar, especialmente con relación a aspectos psicosociales, que involucran el estado anímico, la autonomía, la aceptación social y la percepción de los recursos económicos familiares. Las altas prevalencias de sobrepeso y obesidad registradas constituyen un componente más en el abanico de carencias de estas poblaciones, que agudizan y reproducen su condición de pobreza y vulnerabilidad. 


\section{AGRADECIMIENTOS}

Las autoras agradecen a los supervisores, directores, docentes, niños y niñas de las escuelas participantes en este estudio y a la Lic. Antonella Bazán por asistir a las y los escolares durante la realización de la encuesta de calidad de vida relacionada a la salud. Este trabajo se realizó en el marco de una beca doctoral otorgada por el Consejo Nacional de investigaciones Científicas y Técnicas (Res No 4354, año 2013).

\section{REFERENCIAS BIBLIGRÁFICAS}

1. Sen A. Desarrollo y libertad. Buenos Aires: Planeta; 2000.

2. Ferullo H. Sobre los conceptos de pobreza y subdesarrollo en el pensamiento. En: Bolsi A, Paolasso P, (eds.). Geografía de la pobreza en el Norte Grande Argentino. San Miguel de Tucumán: CONICET, PNUD, Universidad Nacional de Tucumán; 2009. p. 41-52.

3. Longhi F. La tasa de miseria de los hogares: Una aproximación metodológica y conceptual a la medición, distribución espacial y variaciones de la pobreza extrema en Argentina durante la década del noventa. Estudios Geográficos. 2011;72(271):505-533.

4. Moreno M. La pobreza: una medición en busca de su contenido conceptual. En: III Jornadas Argentinas de Estudios de Población; Santa Rosa; 1995.

5. Lawrence Aber J, Bennett N, Conley D, Li J. The effects of poverty on child health and development. Annual Review of Public Health. 1997;18(1):463-483.

6. Rajmil L, Díez E, Peiró R. Desigualdades sociales en la salud infantil: informe SESPAS 2010. Gaceta Sanitaria. 2010;24(Suppl1):42-48.

7. Sepúlveda D, Álvarez L. Exclusión social y calidad de vida relacionada con la salud en personas entre 25 y 60 años de la zona nororiental de Medellín-Colombia. Revista Facultad Nacional de Salud Pública. 2012;30(1):45-56.

8. Organización Mundial de la Salud. Informe de la Comisión para acabar con la obesidad infantil [Internet]. Ginebra: OMS; 2016 [citado 10 dic 2016]. Disponible en: https://goo.gl/piHhsJ.

9. Peña M, Bacallao J. La obesidad en la pobreza: un problema emergente en las Américas. Washington DC: OPS; 2000.
10. Figueroa Pedraza D. Obesidad y pobreza: marco conceptual para su análisis en Latinoamérica. Saúde e Sociedade. 2009;18(1):103-117.

11. Bolzán A, Mercer R, Ruiz V, Brawerman J, Marx J, Adrogué G, Carioli N, Cordero C. Evaluación nutricional antropométrica de la niñez pobre del norte argentino: proyecto encuNa. Archivos Argentinos de Pediatría. 2005;103(6):545-555.

12. Mercer R, Bolzán A, Ruiz V, Brawerman J, Marx J, Adrogué G, Carioli N, Cordero C. Encuesta de nutrición de la niñez del norte argentino: proyecto encuNa-Parte II; el estado nutricional y el contexto familiar y social. Archivos Argentinos de Pediatría. 2005;103(6):556-565.

13. Bolsi A, Paolasso P, editores. Geografía de la pobreza en el Norte Grande Argentino. San Miguel de Tucumán: CONICET, PNUD, Universidad Nacional de Tucumán; 2009.

14. Ministerio de Salud. Encuesta nacional de nutrición y salud: documento de resultados [Internet]. Buenos Aires: Ministerio de Salud; 2007 [citado 10 dic 2016]. Disponible en: https://tinyurl.com/ y97cfwez.

15. Kovalskys I, Corvalán C, Chamorro V, Casini V, Weisstaub G, Recalde A. Factores obesogénicos vinculados con el ambiente infantil. En: Uauy R, Carmuega E, (eds.). Crecimiento saludable: entre la desnutrición y la obesidad en el Cono Sur. Buenos Aires: INTA, CESNI, Instituto Danone; 2012. p. 175-205.

16. Aguirre P. Pobres gordos, ricos flacos: la alimentación en crisis. Buenos Aires: Capital Intelectual; 2004

17. Calvo E. Evaluación del estado nutricional de niñas, niños y embarazadas mediante antropometría. Buenos Aires: Ministerio de Salud de la Nación; 2009.

18. Montero A. Evolución de la epidemiología de la obesidad en los países emergentes. En: Formiguera X. Obesidad: un reto sanitario de nuestra civilización. Barcelona: Fundación Medicina y Humanidades Médicas; 2004.

19. Rajmil L, López-Aguilà S, Mompart-Penina A. Calidad de vida relacionada con la salud y factores asociados al sobrepeso y la obesidad en la población infantil de Cataluña. Medicina Clínica. 2011;137(2):37-41.

20. Shumaker S, Naughton M. The international assessment of health-related quality of life: a theoretical perspective. En: Shumaker S, Berzon R. The international assessment of health-related quality 
of life: theory, translation, measurement and analysis. Oxford: Rapid Communications; 1995.

21. Pane S, Solans M, Gaite L, Serra-Sutton V, Estrada MD, Rajmil, L. Instrumentos de calidad de vida relacionada a la salud en la edad pediátrica: revisión sistemática de la literatura, actualización. Barcelona: Agencia de Evaluación de Tecnología e Investigación Médicas; 2006.

22. Roizen M, Figueroa C, Salvia L. Calidad de vida relacionada con la salud en niños con enfermedades crónicas: comparación de la visión de los niños, sus padres y sus médicos. Archivos Argentinos de Pediatría. 2007;105(4):305-313.

23. Berra S, Bustingorry V, Henze C, Díaz $M$, Rajmil L, Butinof M. Adaptación transcultural del cuestionario KIDSCREEN para medir la calidad de vida relacionada con la salud en población argentina de 8 a 18 años. Archivos Argentinos de Pediatría. 2009;107(4):307-314.

24. Mamondi V. Sobrepeso, obesidad y calidad de vida relacionada con la salud en escolares de Bahía Blanca. Córdoba: Universidad Nacional de Córdoba, Facultad de Ciencias Médicas, Escuela de Salud Pública; 2011.

25. Instituto Nacional de Estadísticas y Censos. Índice de privación material de los hogares: total por fracción censal, Tucumán, año 2001 [Internet]. Buenos Aires: INDEC [citado 5 ene 2017]. Disponible en: https://tinyurl.com/y9cqup9o.

26. Madariaga H. Características de los hogares pobres del Norte Grande Argentino: su análisis empleando sistemas de información geográfica [Internet]. VII Jornadas Argentinas de Estudios de Población; Tandil; 2005 [citado 10 ene 2017]. Disponible en: https://tinyurl.com/y8wf5ep4.

27. Instituto Nacional de Estadísticas y Censos. Porcentaje de población con necesidades básicas insatisfechas por radio censal, Tucumán, años 2001/2010 [Internet]. Buenos Aires: INDEC [citado 5 ene 2017]. Disponible en: https://tinyurl. com/y8tcfvjl.

28. Instituto Nacional de Estadísticas y Censos. Porcentaje de población en área urbana y rural por radio censal, Tucumán, año 2010 [Internet]. Buenos Aires: INDEC [citado 5 ene 2017]. Disponible en: https://tinyurl.com/mwfrsxw.

29. Zuccardi R, Fadda G. Bosquejo agrológico de la provincia de Tucumán. San Miguel de Tucumán: Universidad Nacional de Tucumán, Facultad de Agronomía y Zootecnia; 1985.

30. Provincia de Tucumán, Sistema de Información Geográfica. Inundaciones por departa- mento, Provincia de Tucumán, año 2000, 2001, 2002 y 2007 [Internet]. San Miguel de Tucumán: IDE [citado 5 nov 2016]. Disponible en: https:// tinyurl.com/ycfc7b3b.

31. La Nación. Trágico temporal en el sur de Tucumán [Internet]. La Nación; 13/02/2001 [citado 5 nov 2016]; sec. Información General. Disponible en: https://tinyurl.com/ybut9j32.

32. La Gaceta. El desborde del río Chico dejó aislado a Niogasta [Internet]. La Gaceta; 22/03/2006 [citado 5 nov 2016]; sec. Información General. Disponible en: https://tinyurl.com/ya928y72.

33. La Gaceta. A cuatro meses de las inundaciones, todavía quedan familias evacuadas [Internet]. La Gaceta; 22/09/2016 [citado 5 nov 2016]; sec. Sociedad. Disponible en: https://tinyurl.com/ yawrh2gv.

34. Bolsi A. Población, azúcar e industria rural en Tucumán, Argentina. Geographicalia. 2000;38(1): 85-109.

35. Morales Solá H. La feria de Simoca. San Miguel de Tucumán: Municipalidad de Simoca; 2010.

36. Instituto Nacional de Estadística y Censos. Provincia de Tucumán: población ocupada en viviendas particulares por categoría ocupacional y sector en el que trabaja, según sexo y máximo nivel de instrucción alcanzado; año 2010 [Internet]. Buenos Aires: INDEC; 2010 [citado 5 ene 2017]. Disponible en: https://tinyurl.com/ ybvxy69h.

37. Juárez E, Rodríguez C. Dinámica del crecimiento y de la redistribución de la población en la provincia de Tucumán (1980-2010). En: Ortiz de D'Arterio JP, (ed.). Transformaciones socioterritoriales y calidad de vida en Tucumán. San Miguel de Tucumán: Universidad Nacional de Tucumán, Facultad de Filosofía y Letras; 2015.

38. Ministerio de Educación de la Provincia de Tucumán. Evolución de la matrícula total provincia Tucumán (1999-2016) [Internet]. San Miguel de Tucumán: Ministerio de Educación de la Provincia de Tucumán; 2015 [citado 10 dic 2016]. Disponible en: https://tinyurl.com/y988fu9o.

39. Comité Nacional de Crecimiento y Desarrollo. Guías para la evaluación del crecimiento físico [Internet]. Buenos Aires: Sociedad Argentina de Pediatría; 2013 [citado 10 dic 2016]. Disponible en: https://tinyurl.com/ydbd4ovb.

40. World Health Organization. WHO AnthroPlus for personal computers manual: software for assessing growth of the world's children and ado- 
lescents [Internet]. Geneva: WHO; 2009 [citado 10 dic 2016]. Disponible en: https://tinyurl.com/ yb8cqntu.

41. Berra $S$, Tebé $C$, Esandi $M$, Carignano C. Fiabilidad y validez del cuestionario KIDSCREEN-52 para medir calidad de vida relacionada con la salud para población argentina de 8 a 18 años. Archivos Argentinos de Pediatría. 2013;111(1):29-35.

42. The KIDSCREEN Group Europe. The KIDSCREEN questionnaires: quality of life questionnaires for children and adolescents. Lengerich: Pabst Science Publishers; 2006.

43. Cerda J, Vera C, Rada G. Odds ratio: aspectos teóricos y prácticos. Revista Médica de Chile. 2013;141(10):1329-1335.

44. Poletti O, Barrios L. Obesidad e hipertensión arterial en escolares de la ciudad de Corrientes, Argentina. Archivos Argentinos de Pediatría. 2007;105(4):293-298.

45. Oyhenart EE, Dahinten SL, Alba JA, Alfaro EL, Bejarano IF, Cabrera GE, et al. Estado nutricional infanto juvenil en seis provincias de argentina: variación regional. Revista Argentina de Antropología Biológica. 2010;10(1):5-62.

46. Padilla IS. Prevalencia de sobrepeso-obesidad y factores asociados con valor predictivo-preventivo en escolares de 6 a 11 años de Río GaIlegos, Santa Cruz, Argentina. Salud Colectiva. 2011;7(3):377-388.

47. Cesani MF, Garraza M, Bergel Sanchís ML, Luis MA, Torres MF, Quintero FA, et al. A comparative study on nutritional status and body composition of urban and rural schoolchildren from Brandsen District (Argentina). PLoS ONE. 2013;8(1):e52792. doi: 10.1371/journal.pone.0052792.

48. Bergel Sanchis ML, Quintero FA, Navazo B, Cesani MF, Garraza M, Torres MF, et al. Caracterización del estado nutricional en relación con factores socioambientales de la población escolar del partido de Punta Indio (provincia de Buenos Aires, Argentina). Revista Argentina de Antropología Biológica. 2016;18(2):1-13.

49. Garraza M, Cesani MF, Navone GT, Oyhenart EE. Malnutrition and body composition in urban and rural schoolchildren: A cross-sectional study in San Rafael, Mendoza (Argentina). American Journal of Human Biology. 2016;28(6):796-803.

50. Shields M, Tremblay MS. Canadian childhood obesity estimates based on WHO, IOTF and CDC cut-points. International Journal of Pediatric Obesity. 2010;5:265-273.
51. Bergel ML, Cesani MF, Cordero ML, Navazo B, Olmedo S, Quintero F, Sardi M, Torres MF, Aréchiga J, Méndez de Pérez, Marrodán MD. Valoración nutricional de escolares de tres países iberoamericanos: análisis comparativo de las referencias propuestas por el International Obesity Task Force (IOTF) y la Organización Mundial de la Salud. Nutrición Clínica y Dietética Hospitalaria. 2014;34(1):8-15.

52. Popkin BM, Adair LS, Ng SW. Global nutrition transition and the pandemic of obesity in developing countries. Nutrition Review. 2012;70(1):3-21.

53. Cesani MF, Castro LE, Luis MA, Torres MF, Quintero FA, Luna ME, Bergel ML, Oyhenart EE. Sobrepeso y obesidad en escolares de Brandsen en relación a las condiciones socio-ambientales de residencia. Archivos Argentinos de Pediatría. 2010;108(4):294-302.

54. Aguirre P. Aspectos socioantropológicos da obesidade na pobreza. En: Peña M, Bacallao J. Obesidade e pobreza: um novo desafio à saúde pública. São Paulo: Roca; 2006. p. 12-26.

55. Longhi F. Sistemas de información geográfica y desnutrición infantil en el Norte Grande Argentino. Revista Latinoamericana de Población. 2012;10(1):77-101.

56. Ottova V, Erhart M, Rajmil L, Dettenborn-Betz $\mathrm{L}$, Ravens-Sieberer U. Overweight and its impact on the health-related quality of life in children and adolescents: results from the European KIDSCREEN survey. Quality of Life Research. 2012;21(1):59-69.

57. Hurtado-Valenzuela JG, Álvarez-Hernández G. Calidad de vida relacionada con la salud del niño y del adolescente con obesidad. Salud Mental. 2014;37(2):19-125.

58. Rodríguez Barrera JC, Bastidas M, Genta G, Olaya-Contreras $\mathrm{P}$. Calidad de vida percibida por los escolares con sobrepeso y obesidad, de sectores populares de Medellín, Colombia. Universitas Psychologica. 2016;15(2):301-314.

59. Villaseñor Bayardo S, Ontiveros Esqueda C, Cárdenas Cibrián K. Salud mental y obesidad. Investigación en Salud. 2006;8(2):86-90.

60. Figueroa M, Contini N, Lacunza A, Levín M, Suedan Estévez A. Las estrategias de afrontamiento y su relación con el nivel de bienestar psicológico: Un estudio con adolescentes de nivel socioeconómico bajo de Tucumán (Argentina). Anales de Psicología. 2005;21(1):66-72.

61. Ministerio de Salud de la Nación, Unidades Académicas de Psicología de Universidades Na- 
cionales. Problemáticas de salud mental en la infancia: proyecto de investigación, informe final [Internet]. Buenos Aires: Ministerio de Salud de la Nación; 2007 [citado 5 ene 2017]. Disponible en: https://tinyurl.com/y9dlw8kw.

62. Contini EN, Lacunza AB, Medina SE, Álvarez M, González M, Coria V. Una problemática a resolver: soledad y aislamiento adolescente. Revista Electrónica de Psicología Iztacala. 2012;15(1):127-149.

63. Morison P, Masten A. Peer reputation in middle school as a predictor of adaptation in adolescence: a seven year follow up. Child Development. 1991;62(5):991-1007.

64. Nansel TR, Overpeck M, Pilla RS, Ruan WJ, Simona-Morton B, Scheidt P. Bullying behaviors among US youth: prevalence and association with psychosocial adjustment. JAMA. 2001;285(16):2094-2100.
65. Janssen I, Craig W, Boyce W, Pickett W. Associations between overweight and obesity with bullying behaviors in school-aged children. Pediatrics. 2005;113(5):1187-1194.

66. Galardi Andonegui MS, Ugarte Líbano R. Maltrato entre iguales (bullying) en la escuela: ¿cuál es el papel de los pediatras de atención primaria? Pediatría Atención Primaria. 2005;7(25):11-19.

67. Scutti CS, Yukari Seo G, Serraneto Amadeu R, Ferreira Sampaio R. O enfrentamento do adolescente obeso: a insatisfação com a imagem corporal e o bullying. Revista da Facultade de Ciências Médicas de Sorocaba. 2014;16(3):130-133.

68. Attree P. The social costs of child poverty: A systematic review of the qualitative evidence. Children \& Society. 2006;20:54-66.

\section{FORMA DE CITAR}

Cordero ML; Cesani MF. Sobrepeso, obesidad y salud percibida en contextos de pobreza de Tucumán, Argentina. Salud Colectiva. 2018;14(3):563-578. doi: 10.18294/sc.2018.1309.

Recibido: 31 de enero de 2017 | Versión final: 12 de junio de 2017 | Aprobado: 21 de febrero de 2018 